\title{
From the Eden Model to the Kinetic Growth Walk: A Generalized Growth Model with a Finite Lifetime of Growth Sites
}

\author{
Armin Bunde, ${ }^{1,2}$ Sasuke Miyazima, ${ }^{1,3}$ and H. Eugene Stanley ${ }^{1}$
}

Received December 11, 1986

We propose a new class of cluster growth models where growth sites have a finite lifetime $\tau$, which contains as special cases the Eden model $(\tau=\infty)$ and the kinetic growth walk $(\tau=1)$. For finite but large $\tau$ values the growth process can be characterized by a crossover time $t_{X}$; for times below $t_{X}$ an Eden-type cluster is formed, while for times above $t_{X}$ the growth process belongs to the universality class of the self-avoiding random walk. The crossover time increases monotonically with $\tau$. We develop a scaling theory for the time evolution of the mean end-to-end distance between the seed and the last-added site, and for the average number of growth sites by which the kinetics of the growth process can be characterized. We test this scaling theory by extensive Monte Carlo simulations. We also extend our results to inhomogeneous media (percolation systems).

KEY WORDS: Cluster growth models; Eden model; kinetic growth walk.

\section{INTRODUCTION}

In recent years, considerable attention has been addressed to cluster growth models. Cluster growth models have been used to describe a large variety of spreading phenomena ${ }^{(1-11)}$ ranging from the growth of tumors, epidemics, and forest fires to signal propagation and network mechanics. Also, the formation of diffusion fronts ${ }^{(12,13)}$ and linear and branched

\footnotetext{
${ }^{1}$ Center for Polymer Studies and Department of Physics, Boston University, Boston, Massachusetts 02215.

${ }^{2}$ Fakultät für Physik, Universität Konstanz, D-7750 Konstanz, West Germany.

${ }^{3}$ Department of Engineering Physics, Chubu University, Kasugai, Aichi 487, Japan.
} 
polymers ${ }^{(14)}$ can be conveniently described in terms of cluster growth models. Much effort has been undertaken to investigate the basic growth rules by which the final structure of a cluster and the growth process itself are determined. The final structure can be described by the fractal dimension $d_{f}$, which gives the cluster mass $M$ within a distance $r$ of the seed

$$
M \sim r^{d_{f}}
$$

The growth sites are defined as that part of the cluster surface where the cluster can grow. The dynamics of the growth process can be characterized by the fractal dimension $d_{g}$, which relates $G$, the mass of the growth sites inside radius $r$, to $r$, for $r<R$, where $R$ is the radius of gyration. Thus

$$
\left.G \sim r^{d_{g}}, \quad r<R\right]
$$

It has been shown recently ${ }^{(8)}$ that the "dynamic" exponent $d_{g}$ and the "static" exponent $d_{f}$ are independent of each other. If the next growth site to be tested is from a probability distribution $P(r)$

$$
P(r) \sim r^{-\beta}
$$

where $r$ is the distance from the most recently added cluster site, then for $\beta \geqslant 2$ the growth exponent $d_{g}$ changes continuously with $\beta .^{(7)}$

In this paper we will consider a comparatively simple model where the growth sites are chosen from a random homogeneous distribution, $\beta=0$, of neighbors of already occupied sites. We will consider a novel feature of growth sites, namely the possibility of becoming inactive. We identify cluster sites as "sick" sites, where epidemics can spread. The assumption of a finite lifetime of growth sites is equivalent to the assumption that a "sick" site can infect until now "healthy" sites only for a certain time $\tau$ : i.e., if a neighbor of a site $A$ that was infected at time $t_{1}$ has not been infected by $A$ up to time $\tau+t_{1}$, it cannot be infected after that time by $A$. We find that this quite natural assumption does affect strongly not only the dynamic universality class, but also the static universality class of the growth process. For $\tau=\infty$ we recover the well-known Eden model where $d_{f}=2$ and $d_{g}=1$, while for any finite $\tau$ we find that the growth process belongs to the universality class of self-avoiding random walks (to which also the growth of branched polymers and the kinetic growth walk belong) $d_{f}=4 / 3$ and $d_{g}=0$.

In contrast to these earlier studied growth processes, for which the probability of growing large clusters is strongly reduced and only nonasymptotic values of the fractal dimension are observed, the probability of death of a cluster in our new process is extremely low. Large clusters can be grown easily and $d_{f}=4 / 3$ is clearly observed. 
We also modify our model and allow for immune sites where the cluster cannot grow. Here we find that $d_{f}$ and $d_{g}$ are not changed for finite $\tau$ values for both $p>p_{c}$ and $p=p_{c}$, where $p_{c}$ is the critical concentration of "sick" sites where indefinite growth still can take place. In contrast, when $\tau=\infty$ one has $d_{f}=2$ and $d_{g}=1$ for $p>p_{c}$, while for $p=p_{c}, d_{f}=91 / 48$ and $d_{g} \simeq 3 / 4$. We find that for finite but large $\tau$ values the growth process can be characterized by a crossover time $t_{X}$. For $t<t_{X}$ an Eden cluster is formed, while for $t>t_{X}$ the growth process belongs to the universality class of self-avoiding random walks. We develop a scaling theory for the basic relevant quantities and test it by Monte Carlo simulations.

\section{THE MODEL}

For specificity, consider a square lattice. To describe the spreading of an epidemic, assume that each site of the lattice can either be empty or occupied by three types of particles that we shall call sick (S), immune (I), and growth (G). At time $t=0$ all lattice sites are empty. At time $t=1$ we place an $S$ particle at the origin and occupy the four nearest neighbor sites with $\mathrm{G}$ particles. Sites occupied by $\mathrm{G}$ particles are called growth sites, since they constitute that part of the lattice to which the epidemic can grow. At time $t=2$ one of the $\mathrm{G}$ particles is chosen randomly and converted into an $\mathrm{S}$ particle with probability $p$ and into an I particle with probability $1-p$. If it is converted into an I particle, we choose randomly one of the remaining $\mathrm{G}$ particles and continue the process. If an $\mathrm{S}$ particle was created again, its empty nearest neighbors become $G$ particles and the time is increased by one. The "infection" spreads by the successive conversion of G particles into $\mathrm{S}$ particles and after $t$ time steps a large cluster of $s=t$ sick particles has been formed. This model and modifications of it have been studied in Refs. 6-10. It has been found that introducing space correlations ${ }^{(7,8)}$ or time correlations ${ }^{(10)}$ between growth sites does not lead to a change in the structure of large clusters. At $p=1$ (no immune particles) the model reduces to the much-studied Eden model.

Now we introduce a new feature into this model for epidemics: we assume that a sick particle remains sick forever, but only can infect its neighboring growth particles for a certain time $\tau$. If the particle gets sick at time $t_{1}$, then it can infect its neighboring $\mathrm{G}$ particles only up to time $t_{1}+\tau$. After that time the G particle "dies" and its site becomes empty again.

(i) For $\tau=\infty$, the static properties are in the same universality class as percolation, since the process of randomly converting growth particles into $\mathrm{S}$ particles (with weight $p$ ) or into I particles (with weight $1-p$ ) is the same as for percolation. It is known that $d_{f}=2$ for $p>p_{c}$ and $d_{f}=91 / 48$ for $p=p_{c}$. For details we refer to Ref. 8 . 
(ii) For $\tau=1$ and $p=1$, the cluster grows only on the last-added growth sites. This case reduces to the kinetic growth walk, whose properties are in the same universality class as the self-avoiding random walk ${ }^{(4)}$ (used to model the growth of linear polymers). Consequently, we have $d_{f}=4 / 3$ in this case.

The kinetic properties of the growth process can be characterized by the fractal dimensionality $d_{g}$, defined in (1.2).

(i) For $p=1$ and $\tau=\infty$ the growth sites represent the surface of a compact cluster and therefore $d_{g}=1$.

(ii) For $p=p_{c}$ and $\tau=\infty$ the growth sites more nearly form a fractal cut with the external perimeter and therefore $d_{g}=d_{h}-1=3 / 4$, where $d_{h}=7 / 4$ is the fractal dimensionality of the external perimeter (hull) of the cluster.

(iii) On the other hand, for $p=1$ and $\tau=1$ the number of growth sites is constant (if the growth does not die) and therefore $d_{g}=0$.

The physical question we address here is how both the structure of the final cluster (characterized by $d_{f}$ ) and the structure of the growth sites (characterized by $d_{g}$ ) change when $\tau$ varies from 1 to $\infty$ and how this change depends on the "heterogeneity" of the medium, characterized by the presence of the immune particles.

\section{NUMERICAL SIMULATION AND SCALING THEORY}

In Figs. 1a-1d are shown typical clusters for $p=p_{c}=0.5927, \tau=10$; $p=p_{c}, \tau=80 ; p=1, \tau=10 ; p=1, \tau=80$, respectively. The clusters for $p=p_{c}$ and $\tau=10$ look more like a thick chain, while the clusters with $p=1$ and $\tau=80$ have more similarity with percolation and Eden clusters. For finding the fractal dimensionality $d_{f}$ we have studied as a function of time $t(=s)$ the end-to-end distance $\left[\left\langle r^{2}(t)\right\rangle\right]^{1 / 2}$ between the last added $\mathrm{S}$ site and the seed at the origin of the square lattice. $\left\langle r^{2}(t)\right\rangle$ scales as

$$
\left\langle r^{2}(t)\right\rangle \sim t^{2 / d f}
$$

and therefore by measuring $\left\langle r^{2}(t)\right\rangle$ we can determine $d_{f}$.

\section{1. $p=p_{c}$ (Critical Concentration of Sick Sites)}

Figure 2a shows $\left\langle r^{2}(t)\right\rangle$, averaged over $10^{4}$ runs, as a function of time for $p=p_{c}$ and four values of $\tau$. For small times corresponding to small cluster sizes $\left\langle r^{2}(t)\right\rangle$ is independent of $\tau$ and the slope of the curve of the 


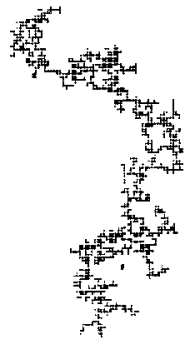

(a)

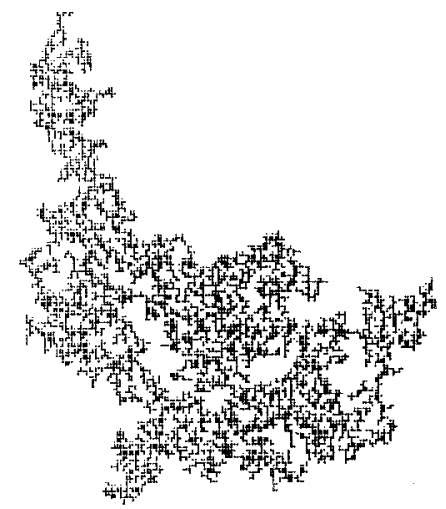

(c)

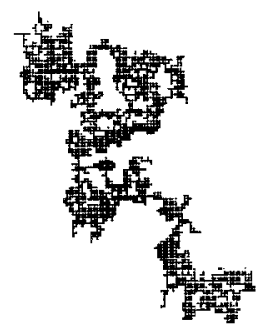

(b)

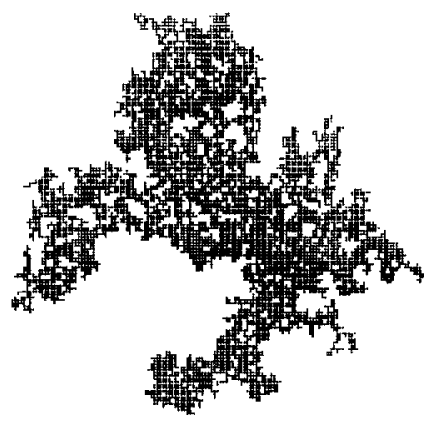

(d)

Fig. 1. Typical clusters for $p=1$ (no immune sites) and $p=p_{c}$ for two values of lifetime $\tau$ of growth sites. (a) $p=p_{c}, \tau=10$. The number $s$ of cluster sites is $s=1000$. (b) $p=p_{c}, \tau=80$; $s=2400$. (c) $p=1, \tau=10 ; s=2000$. (d) $p=1, \tau=80 ; s=6000$.

double logarithmic plot equals 1.05 , the value for percolation clusters. For large times the slope is equal to 1.5 , corresponding to $d_{f}=4 / 3$, the value for self-avoiding random walks. The crossover time $t_{X}$ increases with increasing value of $\tau$.

In Fig. 2b we have plotted, for the same set of $\tau$ values as in Fig. 2a, the number of growth sites averaged over $10^{4}$ runs as a function of time. Again, for small times, all curves coincide, and the slope of $G(t)$ in the double logarithmic plot is equal to 0.4 , corresponding to $d_{g}=0.75$, the value for percolation. For large times, $G(t)$ reach a plateau and therefore $d_{g}=0$, as for self-avoiding random walks. The height of the plateau $G_{\infty}$ increases with increasing $\tau$; we have found numerically that for large $\tau, G_{\infty}$ follows a power law

$$
G_{\infty} \sim \tau^{\alpha_{g}}
$$




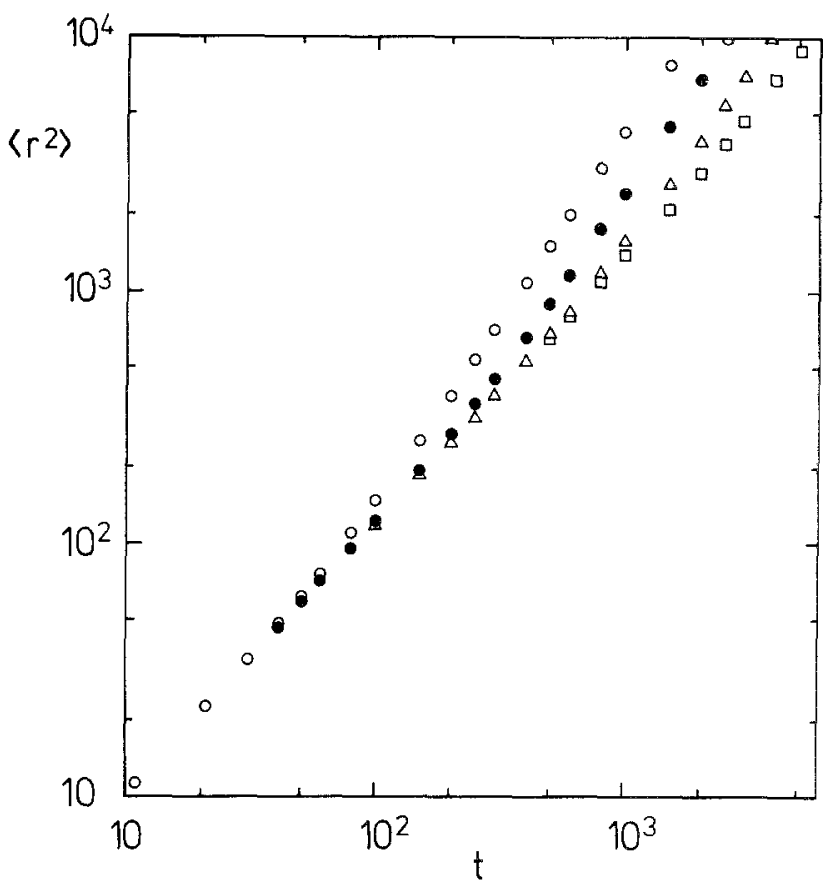

(a)

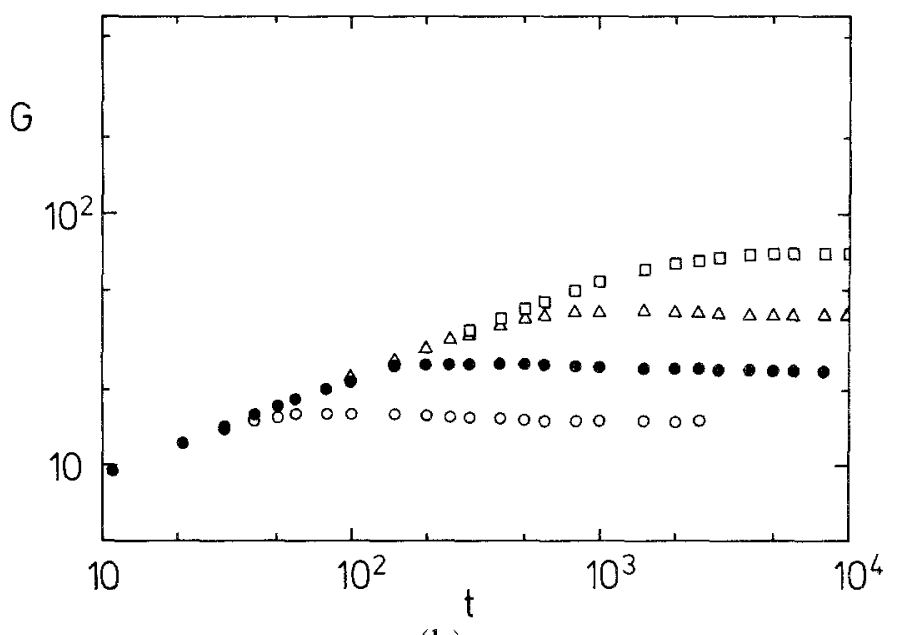

(b)

Fig. 2. Plots of (a) the mean square of the end-to-end distance $\left\langle r^{2}(t)\right\rangle$ and (b) the number of growth sites $G(t)$ versus $t=S$ for clusters at $p=p_{c}$ and various values of $\tau:(0) 20,(0) 40$, $(\triangle) 80,(\square) 160$. For each data point, averages over more than 10,000 clusters have been made. 
where $\alpha_{g}$ is a new exponent, which for $p=p_{c}$. has the value $\alpha_{g} \simeq 0.75$. The crossover time increases when the lifetime $\tau$ of the $G$ particles is increased. In both $\left\langle r^{2}\right\rangle$ and $G$, a crossover from a percolation growth process to a growth walk is observed. Hence the crossover times in the end-to-end distance and in the number of growth sites must be identical

$$
t_{X} \sim \tau^{\phi}
$$

where $\phi$ is a new exponent. In the following we will develop a scaling theory by which the crossover exponent $\phi$ can be related to $\alpha_{g}$, $d_{f}^{\infty} \equiv d_{f}(\tau=\infty)=91 / 48$, and $d_{g}^{\infty} \equiv d_{g}(\tau=\infty) \simeq 3 / 4$. We assume that for large $\tau, G$ and $\left\langle r^{2}\right\rangle$ can be written as

$$
G(t, \tau)=\tau^{\alpha_{g}} \tilde{G}\left(t / t_{X}\right)
$$

and

$$
\left\langle r^{2}(t, \tau)\right\rangle=\tau^{\alpha_{r}} \widetilde{R}\left(t / t_{X}\right)
$$

For small times $\mathrm{t} \ll t_{X}$ the finite lifetime of the $\mathrm{G}$ particles cannot affect the growth of a percolation cluster. Hence both $G$ and $\left\langle r^{2}\right\rangle$ must be independent of $\tau$,

$$
G(t) \sim t^{d_{g}^{\infty} / d_{f}^{\infty}}
$$

and

$$
\left\langle r^{2}(t)\right\rangle \sim t^{d_{f}^{x_{x}}}
$$

Comparing (3.4) with (3.6) and (3.5) with (3.7), we find

$$
\widetilde{G}(x) \sim x^{d_{g}^{\infty} / d_{f}^{\infty}}, \quad x \ll 1
$$

and

$$
\tilde{R}(x) \sim x^{d f_{f}^{\infty}}, \quad x \ll 1
$$

We insert (3.8) into (3.4). Since the result must be independent of $\tau$, we find

$$
\phi=\alpha_{g} d_{f}^{\infty} / d_{g}^{\infty}
$$

Similarly, we obtain for $\alpha_{r}$

$$
\alpha_{r}=\phi \cdot 2 / d_{f}^{\infty}
$$

and therefore $\alpha_{r}$ and $\alpha_{g}$ are related by

$$
\alpha_{r}=2 \alpha_{g} / d_{g}^{\infty}
$$




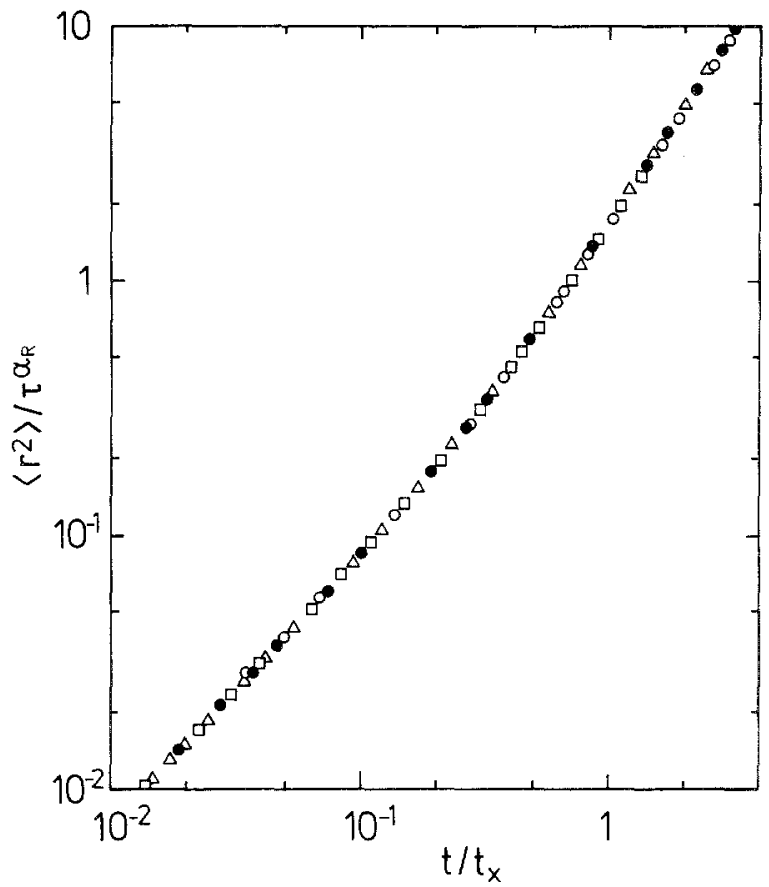

(a)

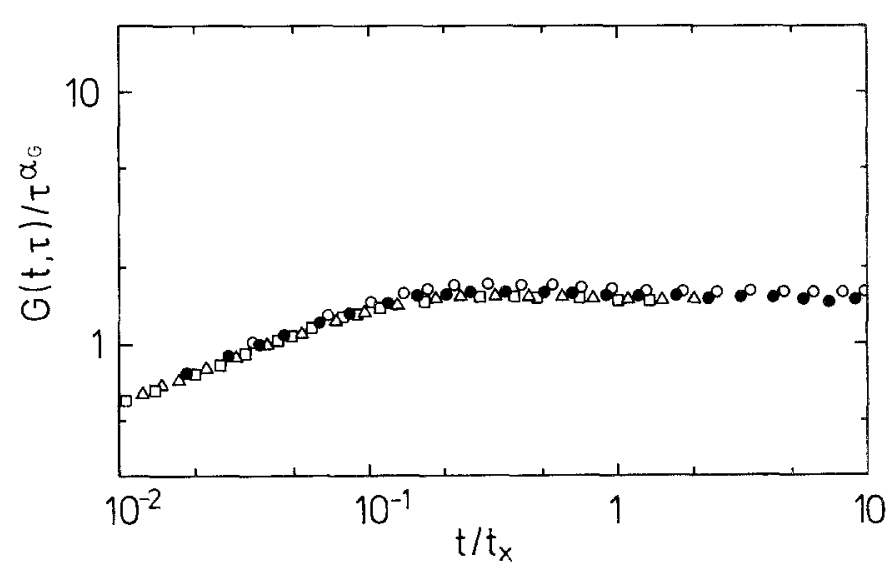

(b)

Fig. 3(a) The mean-square end-to-end distance $\left\langle r^{2}(t)\right\rangle$ and (b) the number of growth sites $G(t)$ for $p=p_{c}$ and $\tau=20,40,80$, and 160 scaled so that all curves collapse upon a single curve. The data collapse supports Eqs. (3.4) and (3.5) with $\alpha_{g}=0.75$. 
Equations (3.10) and (3.12) express the exponents $\alpha_{r}$ and $\phi$ by $\alpha_{g}$ and the known exponents $d_{f}^{\infty}$ and $d_{g}^{\infty}$. A simple and more physical interpretation of (3.11) is as follows: the factor $\tau^{\alpha_{r}}$ in (3.5) can be considered the square of an effective crossover radius $r_{x}$. For $\left\langle r^{2}\right\rangle \ll r_{X}^{2}$, a percolation cluster is grown and hence $\left\langle r^{2}\right\rangle \sim t^{2 / d_{f}^{\infty}}$. For $t=t_{X}$ we have thus $r_{X}^{2} \sim t_{X}^{2 l d f_{f}^{\infty}}$, from which (3.11) follows. In order to test the scaling theory, we have plotted $\left\langle r^{2}(t, \tau)\right\rangle / \tau^{\alpha_{r}}$ and $G(t, \tau) / \tau^{\alpha_{g}}$ as a function of $t / t_{X}$ and have varied the exponent $\alpha_{g}$. We have achieved a data collapse for $\alpha_{g}=0.75 \pm 0.05$ in Fig. 3. So far, we have no explanation for this number, which seems to suggest $\alpha_{g}=d_{g}^{(\infty)}$ or $\alpha_{f}=1 / d_{f}^{\infty}$.

Next we study the growth of clusters in the absence of immune particles, $p=1$.

\section{2. $p=1$ (No Immunity)}

Figures $4 \mathrm{a}$ and $4 \mathrm{~b}$ show the square of the end-to-end distance and number of growth sites as a function of time for four different $\tau$ values. We observe the same qualitative features as for $p=p_{c}$.

Below a crossover time $t_{X}, G$ satisfies the power law (3.6), where now, according to the Eden growth process, $d_{g}^{\infty}=1$ and $d_{f}^{\infty}=2$. Thus, $G \sim t^{1 / 2}$ for $t \ll t_{X}$, which is clearly observed in Fig. $4 \mathrm{~b}$. For $t \gg t_{X}$ we have again $G(t) \sim G_{\infty}=$ const, and hence $d_{g}=0$. We have found that $G_{\infty}$ still satisfies the power law (3.2), but now $\alpha_{g}$ is close to unity, possibly supporting the conjecture $\alpha_{g}=d_{g}^{\infty}$.

Above $t_{X},\left\langle r^{2}(t)\right\rangle$ follows the power law (3.1) with $d_{f}=4 / 3$ and we recover again the characteristic behavior of the self-avoiding random walks as seen in Fig. 4a. Below $t_{X}$, in a large time regime, $\left\langle r^{2}(t)\right\rangle$ is linear in $t$, since Eden clusters are grown with $d=2$. However, for very small $t$ we see some transient behavior, which becomes more pronounced for smaller values of $\tau$. This transient behavior is reminiscent of the situation for the kinetic growth walk $(\tau=1)$. In $\mathrm{KGW}$, the survival probability is extremely low at large times and the transient behavior seems to dominate. This observation led several authors (see, e.g., Ref. 15 and references therein) to the wrong conclusion that the KGW belong to a different universality class than the self-avoiding walk. The same transient behavior occurs in clustergrowth models ${ }^{(15,16)}$ for branched polymers that are chemically linear. ${ }^{(14)}$ In contrast to these, where the asymptotic regime was hard to reach numerically, in our growth model when the lifetime $\tau$ of the G particles is sufficiently large the survival probability of the growth process is high and the asymptotic regime can easily be reached.

We have found in Fig. 5 that for large $\tau\left\langle r^{2}(t, \tau)\right\rangle$ and $G(t, \tau)$ also 


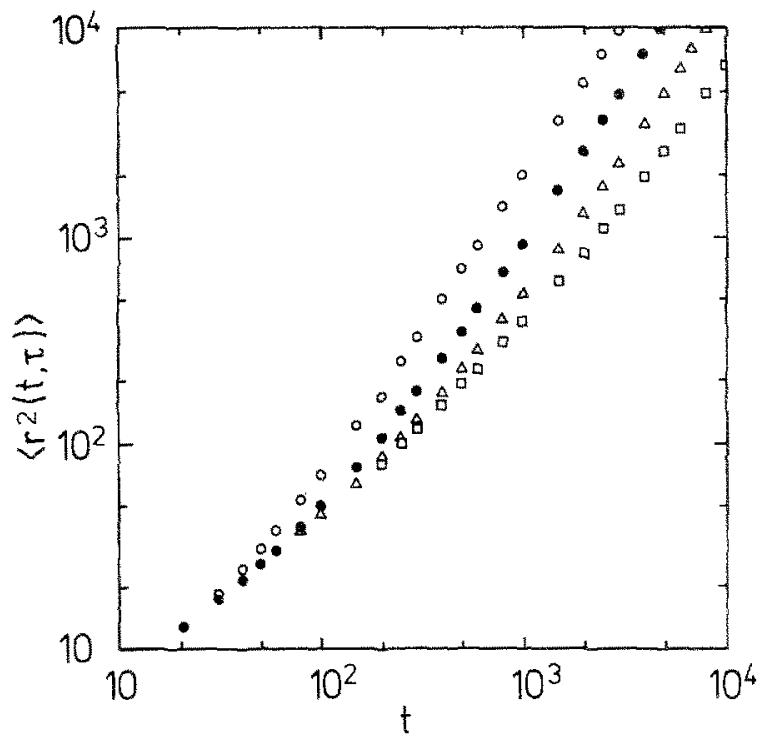

(a)

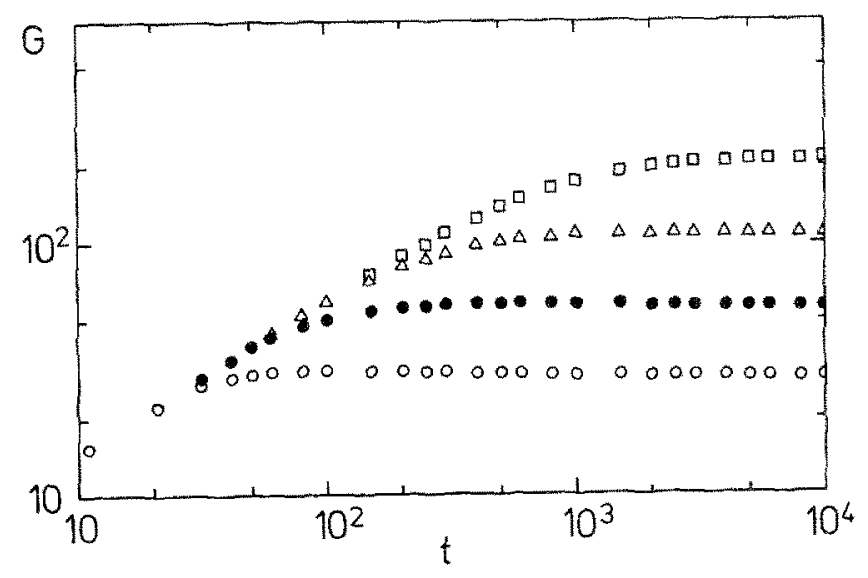

(b)

Fig. 4. Plots of (a) $\left\langle r^{2}(t)\right\rangle$ and (b) $G(t)$ vs. $t$ for $p=1$ and $\tau=(0) 20,(-40,(\Delta), 80$, and (口) 160 . 


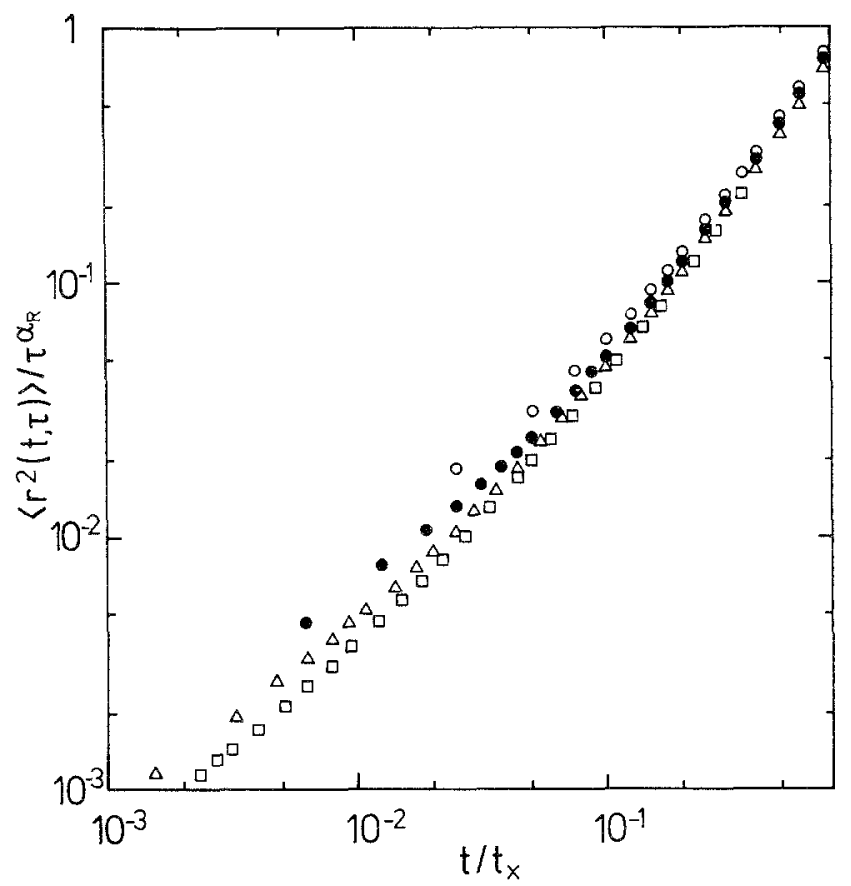

(a)

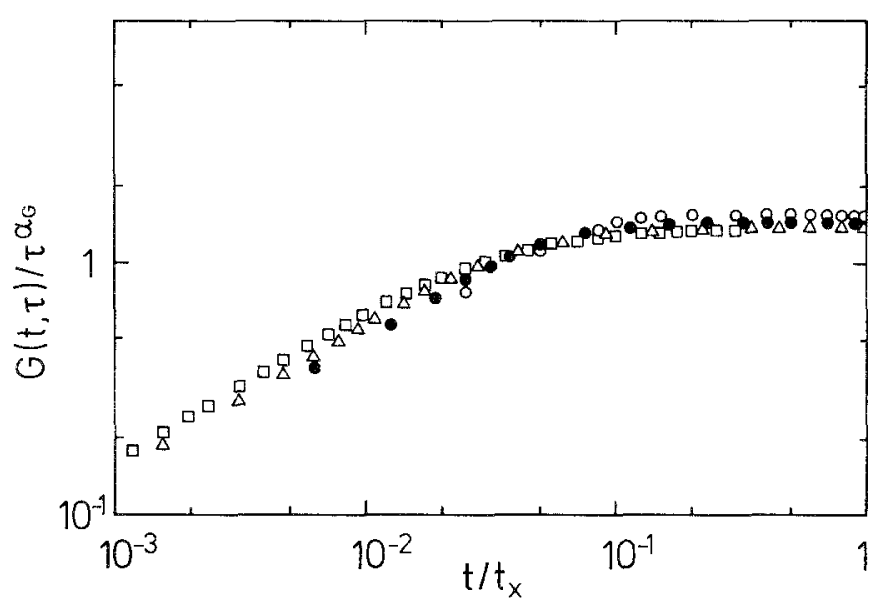

(b)

Fig. 5. Plots of (a) $\left\langle r^{2}(t)\right\rangle$ and (b) $G(t)$ for $p=1$ and $\tau=20,40,80$, and 160 scaled so that all curves collapse upon a single line. The result supports the scaling Ansatz, Eqs. (3.4) and (3.5), with $\alpha_{g}=1$. 
satisfy the scaling relations (3.6)-(3.12), where now $d_{f}^{\infty}=2, d_{g}^{\infty}=1$, and $\alpha_{g} \simeq 1$. For smaller $\tau$ values and short times the scaling does not hold (see above).

\section{3. $p \geqslant p_{c}$ (The Critical Regime)}

In Sections 3.1 and 3.2 we studied the growth process at $p_{c}$ and at $p=1$. We found that for sufficiently large values of $t$ and $\tau$ the end-to-end distance and the number of $G$ particles obeyed scaling laws that are reminiscent of scaling laws close to critical points. The scaling has been described by a new exponent $\alpha_{g}$ which is about 1 at $p=1$ and about $3 / 4$ at $p=p_{c}$. Now we shall study the growth of clusters just above the critical concentration. What do we expect qualitatively to occur? Let us consider first the case of large $\tau$ values and time $t$ well below the crossover time $t_{X}$, where a percolation cluster is grown. On length scales much smaller than the correlation length $\xi \sim\left|p-p_{c}\right|^{-\nu}, v=4 / 3$, the growth process is the same as at $p_{c}$. Therefore we expect that (3.1) and (3.2) hold for $t \ll t_{X}$ and $\left\langle r^{2}(t)\right\rangle \ll \xi^{2}$. By $\left\langle r^{2}\left(t_{X}^{\prime}\right)\right\rangle \sim \xi^{2}$ a second crossover time $t_{X}^{\prime}$ is introduced via $\left\langle r^{2}\left(t_{X}^{\prime}\right)\right\rangle \sim\left(t_{X}^{\prime} d\right)^{2 / d_{f}^{d s}} \sim \xi^{2}$, i.e., $t_{X}^{\prime} \sim \xi_{f}^{d_{f}^{(\infty)}}$. Consequently, we have to distinguish now three time regimes (see Figs. 6 and 7):

1. For $t \ll \xi_{f}^{d_{f}^{\infty}} \ll \tau^{\phi}$ a percolation cluster is grown as at criticality, i.e., $d_{f}^{\infty}=91 / 48$ and $d_{g} \simeq 0.75$.

2. For $\xi_{f}^{d_{j}^{\alpha}} \ll t \ll \tau^{\phi}$, a compact cluster is formed with $d_{f}=2$ and $d_{g}^{\infty}=1$.

3. For $t \gg \tau^{\phi}$ the growth process behaves like a self-avoiding random walk, with $d_{f}=4 / 3$ and $d_{g}=0$.

If $\tau$ is large, but smaller than $\xi^{d_{f}^{\infty}}$, then the situation is similar to that at $p_{c}$ with only one crossover time $t_{X} \sim \tau^{\phi}$. When the concentration $p$ of $S$ particles is enhanced, $\xi$ decreases and the crossover time $t_{X}^{\prime}$ tends to zero. Then the presence of few immune particles is not relevant for the growth process and we expect a situation analogous to $p=1$. To test these predictions, we have studied $\left\langle r^{2}(t, \tau)\right\rangle$ and $G(t, \tau)$ above $p_{c}$ for several concentrations $p$ and four $\tau$ values. Representative examples are shown in Fig. 6, where $p=0.6076$ is fixed and $\tau$ is varied, and Figs. 7, where $\tau=160$ is fixed and $p$ is varied. The figures show clearly the existence of a second crossover time $t_{X}^{\prime}$ which vanishes as $p$ goes to higher values.

\section{CONCLUSION}

In summary, we have introduced a new class of cluster growth models, which are characterized by a finite lifetime $\tau$ of the growth sites. The model 


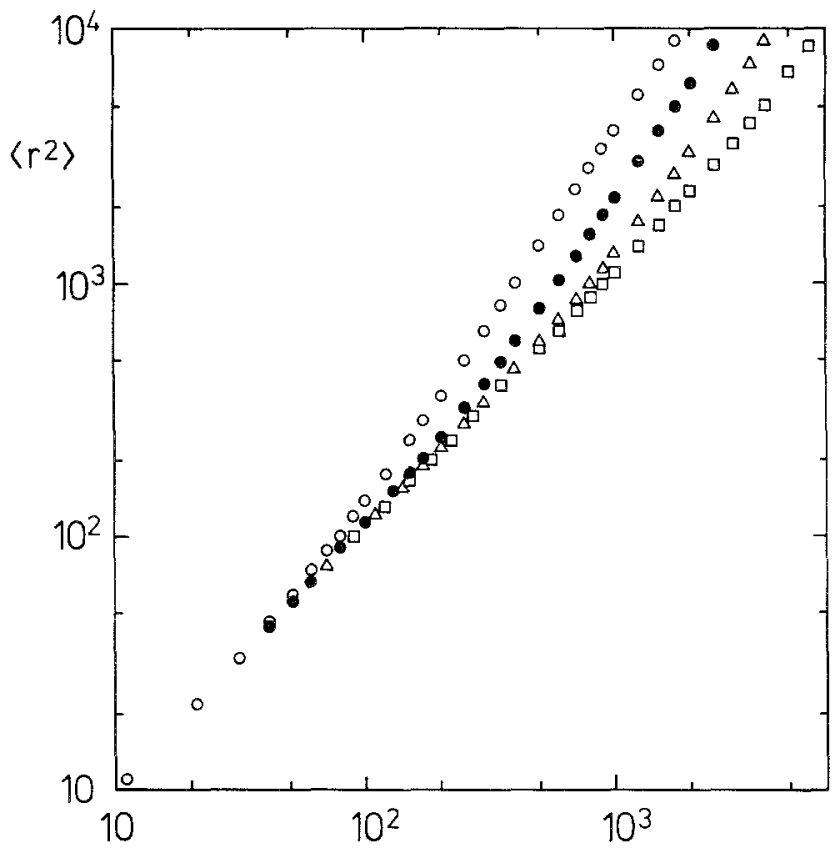

(a)

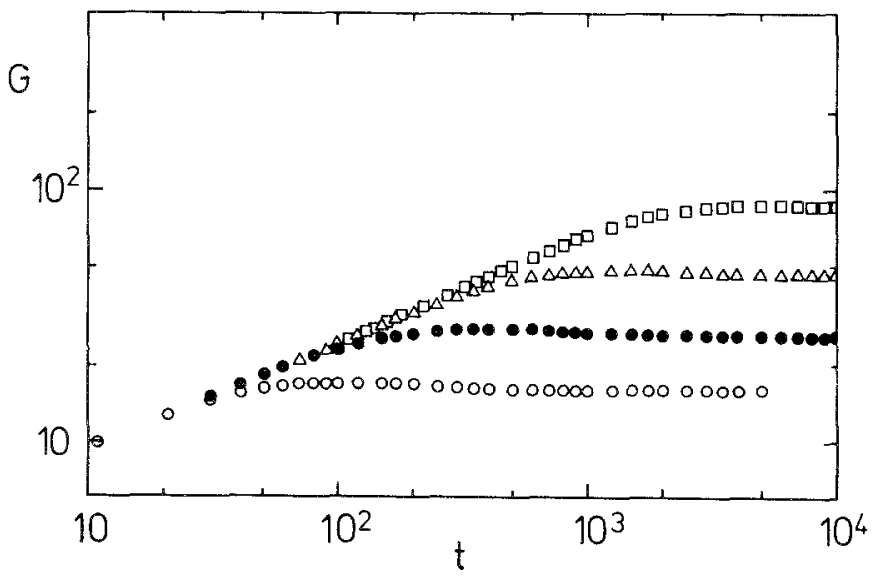

(b)

Fig. 6. Plots of (a) $\left\langle r^{2}(t)\right\rangle$ and (b) $G(t)$ slightly above the critical concentration, $p=0.6076$, for $\tau=20,40,80$, and 160 . 


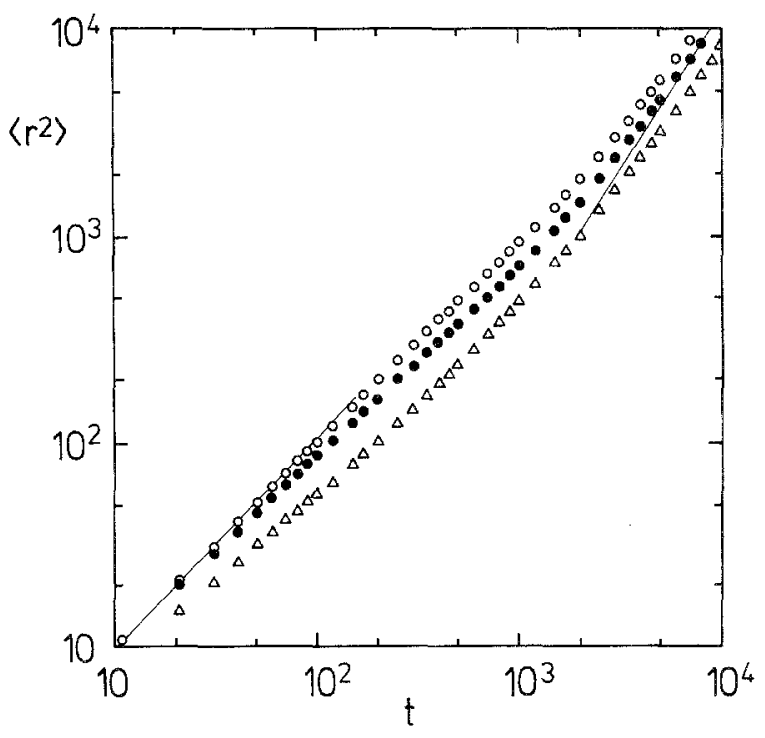

(a)

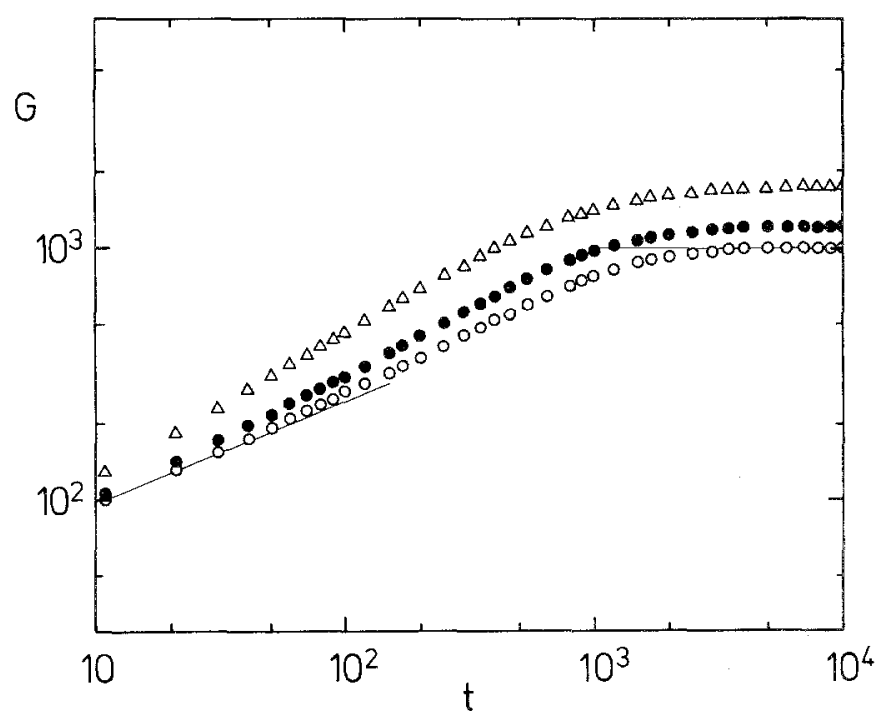

(b)

Fig. 7. Plots of (a) $\left\langle r^{2}(t)\right\rangle$ and (b) $G(t)$ for $\tau=160$ and three values of $p$ above $p_{c}:(O)$ $p=0.622,(\bullet) p=0.655$, and $(\triangle) p=0.8$. 
contains as special cases the kinetic growth walk $(\tau=1)$ and the Eden model $(\tau=\infty)$. We have found that for time $t$ less than a crossover time $t_{X}$, Eden clusters are formed, while for large time $t \gg t_{X}$, the growth process always belongs to the universality class of self-avoiding random walks, with the fractal dimensionality $d_{f}=4 / 3$ and the growth exponent $d_{g}=0$ in two dimensions. For large $\tau$ and in the absence of immune sites, the number of surviving clusters remained practically constant at large times and therefore the fractal dimensionality of the growing clusters was easy to detect numerically. This behavior is in marked contrast to more conventional random walks, such as the kinetic growth walk ${ }^{(15,16)}$ or the growth model for branched polymers, ${ }^{(14)}$ where transient behavior is dominant on a large time scale and thus the asymptotic regime $\left(d_{f}=4 / 3\right)$ is very hard to reach numerically.

It will be interesting to study several variants of our model, where (a) the lifetime of the growth sites is not a constant, but is chosen from a distribution $^{(17)}$ and (b) the growth sites themselves are not chosen randomly in the next time step, but rather from a distribution that depends on the actual distance from growth sites to the last added site (see Ref. 8). Also, we are studying the more general case when growth sites have infinite lifetime with probability $q$ and finite lifetime with probability $1-q$. Preliminary results ${ }^{(17)}$ show that dynamical growth transition occurs at a critical value $q_{c}$. For $q<q_{c}$, the growth process is characterized by $d_{f}=4 / 3, d_{g}=0$, while for $q>q_{c}$, Eden clusters are formed at large times.

\section{ACKNOWLEDGMENT}

We wish to thank J. E. Martin for discussion of a related model under development at Sandia National Lab. We also thank S. Havlin for discussions. This work has been supported by the Deutsche Forschungsgemeinschaft and by a grant from Chubu University. The Polymer Center is supported by grants from NATO, NSF, and ONR.

\section{REFERENCES}

1. G. Deutscher, R. Zallen, and J. Adler, eds., Percolation Structures and Processes (Adam Hilger, Bristol, 1983).

2. F. Family and D. P. Landau, eds. Kinetics of Aggregation and Gelation (North-Holland, Amsterdam, 1984).

3. H. E. Stanley and N. Ostrowsky, eds., On Growth and Form (Martinus Nijhoff, Dordrecht, 1986).

4. H. J. Herrmann, Phys. Rep. 136:153 (1986).

5. M. Eden, in Proceedings Fourth Berkeley Symposium on Mathematical Statistics and Probability, J. Neyman, ed. (University of California Press, Berkeley, 1961), Vol. IV. 
6. P. L. Leath, Phys. Rev. B 14:5046 (1976); Z. Alexandrowicz, Phys. Lett. 80A:284 (1980).

7. A. Bunde, H. J. Herrmann, and H. E. Stanley, J. Phys. A 17:L523 (1985).

8. A. Bunde, H. J. Herrmann, A. Margolina, and H. E. Stanley, Phys. Rev. Lett. 55:653 (1985).

9. F. Family and T. Viscek, J. Phys. $A$ 18:L75 (1985).

10. H. J. Herrmann and H. E. Stanley, Z. Phys. B 60:165 (1985).

11. G. Mackay and N. Jan, J. Phys. A 17:L757 (1985).

12. B. Sapoval, M. Rosso, and J. F. Gouyet, J. Phys. Lett. (Paris) 46:L149 (1985).

13. A. Bunde and J. F. Gouyet, J. Phys. A 18:L285 (1985).

14. S. Havlin, B. Trus, and H. E. Stanley, Phys. Rev. Lett. 53:1288 (1984).

15. I. Majid, N. Jan, A. Coniglio, and H. E. Stanley, Phys. Rev. Lett. 55:2092 (1985).

16. K. Kremer and J. W. Lyklema, Phys. Rev. Lett. 55:2091 (1985).

17. S. Miyazima, A. Bunde, S. Havlin, and H. E. Stanley, J. Phys. A 17:L1159 (1986). 